\title{
REGULACIÓN LEGAL SOBRE LA CONTAMINACIÓN SONORA PRODUCIDA POR LOS MEDIOS DE TRANSPORTE PÚBLICO Y PRIVADO EN LA CIUDAD DE JULIACA
}

\author{
FRANCISCO JÁUREGUI HUAYAPA*
}

\section{RESUMEN}

La presente investigación tuvo como objetivo, determinar la normatividad existente para evitar la contaminación sonora producida por los medios de transporte público y privado en la ciudad de Juliaca, esto a través de un análisis minucioso para determinar la norma específica y adecuada a tal situación; y conforme a ello, proponer alternativas de solución que eviten la contaminación sonora y por ende sus efectos en la sociedad en conjunto. El método a utilizar en la presente investigación será la observación.

PALABRAS CLAVE: Contaminación sonora, decibel, Derecho ambiental, estándares de calidad ambiental, transporte privado, transporte público.

\section{ABSTRACT}

The present investigation had an objective, to determine the existing regulations to prevent the noise pollution produced by public and private transport in the city of Juliaca, through a careful analysis to determine the specific and appropriate to this situation standard; and accordingly, to propose solutions to avoid noise pollution and its effects on society as a whole. The method to utilize in this present investigation will be in observation.

KEY WORDS: Noise pollution, environmental law, environmental quality standards, private transportation, public transportation.

\section{INTRODUCCIÓN}

La contaminación ambiental es un tema que está tomado importancia, por lo cual se han expedido normas para reducir el impacto sobre el medio ambiente de todo tipo de contaminantes, algunos con mayores incidencias, como es el caso de la minería; sin embargo, uno de los tipos de contaminación, lo constituye la contaminación acústica o sonora, esto debido al crecimiento acelerado demográfico, así como al progreso tecnológico, produciendo la alteración del medio ambiente llegando en algunos casos a atentar contra la propia salud de las personas que conviven entre sí.

Actualmente, convivimos en una ciudad totalmente contaminada, que viene tornándose en una situación sin remedio, si no se toma las medidas correctivas y restrictivas para evitar esta forma de contaminación que a futuro solo traerán perjuicio a la sociedad en su conjunto. Frente a ello se alza el problema: ¿Cuál es la regulación legal sobre la contaminación sonora producida por los medios de transporte público y privado en la ciudad de Juliaca?.

No se trata de que exista una cierta incompatibilidad absoluta entre el desarrollo tecnológico, el avance de la población y el mantenimiento del equilibrio ecológico, sino más bien, resulta importante que el hombre sepa convivir en forma armónica con todos los medios tecnológicos que usamos y proteger la salud de las personas.

* Bachiller de la Facultad de Ciencias Jurídicas y Políticas Escuela Profesional de Derecho de la UNA- PUNO. 
De este modo, la justificación jurídica de la presente investigación se encuentra respaldada por la preocupación de establecer la regulación existente sobre la contaminación sonora en nuestro país, y de qué manera afecta a la población usuaria la contaminación sonora producida por los medios de transporte público. Finalmente este trabajo de investigación persigue alcanzar objetivos de importante relevancia, habida cuenta que permitirá formular alternativas de solución para subsanar éstas deficiencias detectadas y evitar la contaminación sonora que se viene dando cada día, garantizándose un servicio de transporte sano y beneficioso para un ambiente libre de contaminación.

\section{OBJETIVOS}

\subsection{Objetivo general}

Determinar la normatividad existente sobre la contaminación sonora producida por los medios de transporte público y privado en la ciudad de Juliaca.

\subsection{Objetivos específicos}

Determinar los niveles legales de sonido permitidos para los medios de transporte público y privado.

Establecer de qué manera afecta a la población la contaminación sonora producida por los medios de transporte público y privado.

\section{MATERIALES Y MÉTODOS}

\subsection{Diseño de investigación}

La presente investigación es cualitativa, en razón de que se procederá mediante las técnicas del análisis y la observación a revisar doctrina, normas acerca de la regulación para evitar la contaminación sonora producida por los medios de transporte público y privado.

El tipo de investigación jurídica corresponde al jurídico-social, puesto que el derecho se presenta en sociedades humanas por cuanto es una institución nacida dentro de ella. En el presente caso, la finalidad será precisar la regulación jurídica sobre la contaminación sonora producida por los medios de transporte público y privado.

El ámbito de estudio se concentra en la legislación y normatividad existente sobre la regulación, para evitar la contaminación sonora aplicada tanto a los medios de transporte público y privado.

\subsection{Universo y muestra}

\subsubsection{Universo}

La unidad de análisis de investigación se ubica en la ciudad de Juliaca, específicamente en los entes encargados de regular el funcionamiento, circulación y control de las unidades de transporte público y privado.

\subsubsection{Muestra}

Para tener resultados explícitos y específicos respecto a la investigación, se constituirá como muestra: la legislación, la normatividad a nivel nacional, y la norma referida a la ciudad de Juliaca que verse sobre materia ambiental de transporte público y privado.

\section{RESULTADOS Y DISCUSIÓN TEÓRICA}

\subsection{La contaminación sonora}

La contaminación acústica es considerada por la mayoría de la población de las grandes ciudades como un factor medioambiental muy importante, que incide de forma principal en su calidad de vida. La contaminación ambiental urbana o ruido ambiental es una consecuencia directa no deseada de las propias actividades que se desarrollan en las grandes ciudades. La contaminación acústica perturba las distintas actividades comunitarias, interfiriendo la comunicación hablada, base esta de la convivencia humana, perturbando el sueño, el descanso y la relajación, impidiendo la concentración y el aprendizaje, y lo que es más grave, creando estados de cansancio y tensión que pueden degenerar en enfermedades de tipo nervioso y cardiovascular (Fonseca Tapia, 2010).

Quizás con menor repercusión a nivel mundial, encontramos la contaminación acústica, demasiado 
frecuente en las ciudades. A partir de los 80 decibelios el oído puede resultar dañado, con menor ruido puede también dañarse si nos exponemos durante un prolongado período. Naturalmente, también debemos denunciar el exceso de ruido, si este es ilegal y en caso de que no haya leyes al respecto, solicitar que sean elaboradas (Flores, Dominguez, \& Sanchez, 1998).

El término contaminación acústica según (Chaname Orbe, 2009), hace referencia al ruido cuando éste se considera como un contaminante, es decir, un sonido molesto que puede producir efectos fisiológicos y psicológicos nocivos para una persona o grupo de personas.

La causa principal de la contaminación acústica es la actividad humana; el transporte, la construcción de edificios y obras públicas, la industria, entre otras. Los efectos producidos por el ruido pueden ser fisiológicos, como la pérdida de audición, y psicológicos, como la irritabilidad exagerada. El ruido se mide en decibelios ( $d B$ ); Un informe de la ORGANIZACIÓN MUNDIAL DE LA SALUD (OMS), considera los $50 \mathrm{~dB}$ como el límite superior deseable.

Existe documentación sobre las molestias de los ruidos en las ciudades desde la antigüedad, pero es a partir del siglo pasado, como consecuencia de la Revolución Industrial, del desarrollo de nuevos medios de transporte y del crecimiento de las ciudades cuando comienza a aparecer realmente el problema de la contaminación acústica urbana. Las causas fundamentales son, entre otras, el aumento espectacular del parque automovilístico en los últimos años y el hecho particular de que las ciudades no habían sido concebidas para soportar los medios de transporte, con calles angostas y firmes poco adecuados.

\subsection{Decibel}

Conforme al D.S. N 085-2003-PCM, es la unidad adimensional usada para expresar el logaritmo de la razón entre una cantidad medida y una cantidad de referencia. El decibel, es usado para describir niveles de presión, potencia o intensidad sonora.

\subsection{Ruido}

Es aquel sonido no deseado, que moleste perjudique 0 afecte a la salud de las personas. (Chaname Orbe, 2009).

Técnicamente, el ruido es un tipo de energía secundaria de los procesos 0 actividades que se propagan en el ambiente en forma ondulatoria compleja, desde una fuente que la genera (foco productor), trasladándose por un medio llamado atmósfera, hasta llegar al receptor a una velocidad determinada y disminuyendo su intensidad cuanto mayor es la distancia y las dificultades del entorno físico. (OEFA, 2011, pág. 5).

\subsubsection{Tipos de Ruido}

Es muy importante identificar todos los tipos posibles de fuentes que están presentes en la ciudad, para una mejor comprensión de los reales riesgos a los que está siendo sometida la población. Los principales focos de ruido ambiental se pueden clasificar en:

4.3.1.1. Circulación de vehículos: De todos los focos de ruido presentes en la ciudad, el tránsito de vehículos destaca en relación a otras fuentes, debido a su presencia generalizada en todo el núcleo urbano.

Además, se pueden encontrar diversas fuentes de ruido en un mismo vehículo: el ruido de la carrocería, el tubo de escape, el motor y hasta el ruido producido por el roce del neumático con la calzada.

También se debe considerar el aumento progresivo del parque automotriz, lo que no va acompañado con un desarrollo de los avances tecnológicos que permitan fabricar autos más silenciosos. Todo esto se ve acrecentado por el mal uso de bocinas, la eliminación de silenciadores en las motos y un mal estado de las máquinas por falta de mantención. Otro factor no menor, es el mal estado de las calzadas y el tipo de material.

4.3.1.2. Carreteras: El aumento de vehículos en las ciudades ha provocado el colapso de las vías urbanas y la consecuente construcción de nuevas vías para descongestionar los principales accesos. No obstante, esta situación ha ocasionado el origen de 
una nueva fuente de ruido en zonas que presentaban menores flujos vehiculares.

4.3.1.3. Aeropuertos: Dentro de los ruidos más molestos están los aviones. Su impacto no sólo afecta zonas directas, sino que también incide sobre zonas aledañas. A esto hay que sumar que el ruido provocado por aviones se agrava por la actividad propia del aeropuerto.

4.3.1.4. Industria: Los procesos productivos conllevan altos niveles de ruido, afectando tanto a los trabajadores como a la población aledaña. En muchos casos, los problemas de ruido se originan debido a la expansión urbanística y al acercamiento de las zonas habitadas a las áreas industriales.

Desde el punto de vista de la comunidad, las instalaciones industriales pueden afectar por el ruido que producen hacia el exterior de sus recintos. Los niveles de este ruido ambiental en la mayoría de los casos son bastante inferiores a $80 \mathrm{~dB}(\mathrm{~A})$, por tanto no constituyen riesgo de daño directo a la audición. $\mathrm{Su}$ efecto perjudicial es fundamentalmente de carácter psicofisiológico, expresándose en la interferencia en actividades que requieren un cierto grado de atención o concentración mental 0 situaciones de descanso. Estas interferencias se producen en general en locales cerrados y el parámetro a considerar es el nivel sonoro de emisión en el espacio receptor. De ahí que la legislación vigente en la Región Metropolitana regule tanto de día como de noche, o bien los niveles máximos admisibles frente a las fachadas de las edificaciones tanto de la propia industria como de los inmuebles afectados.

En las industrias podemos detectar diversos tipos de fuentes, tales como motores, ventiladores, grupos electrógenos, grupos frigoríficos, extractores de humo, bombas de calor, tráfico vehicular propio de la industria, maquinaria, etc. En el caso de la pequeña y mediana industria y los talleres, su ubicación es dispersa en toda la ciudad, incluso, se localizan en viviendas u otros usos sociales, cuya interferencia no es sólo por el ruido radiado al ambiente exterior sino que trasmitido estructuralmente a las viviendas vecinas.
4.3.1.5. Locales Públicos: Los locales de recreación y de diversión, casi siempre, están relacionados a actividades ruidosas. Pubs, discotecas, bares con música, fiestas, restoranes, están asociados a ruidos nocturnos.

4.3.1.6. Construcción: El incremento de las actividades industriales, ha aumentado el desarrollo de las obras públicas. Sus molestias se producen casi siempre durante el día y están asociadas a la utilización de maquinaria pesada.

4.3.1.7. Actividad Humana: La propia actividad humana es una fuente que contribuye a través de labores cotidianas y de recreación, aunque en menor medida, a elevar el nivel sonoro en las ciudades.

De acuerdo a la NTP ISO 1996-1 existen varios tipos de ruido. Sin embargo, para efectos de la presente investigación, se considerarán los siguientes:

\subsubsection{En función al tiempo}

4.3.2.1. Ruido Estable: El ruido estable es aquel que es emitido por cualquier tipo de fuente de manera que no presente fluctuaciones considerables (más de $5 \mathrm{~dB}$ ) durante más de un minuto. Ejemplo: ruido producido por una industria o una discoteca sin variaciones.

4.3.2.2. Ruido Fluctuante: El ruido fluctuante es aquel que es emitido por cualquier tipo de fuente y que presentan fluctuaciones por encima de $5 \mathrm{~dB}$ durante un minuto. Ejemplo: dentro del ruido estable de una discoteca, se produce una elevación de los niveles del ruido por la presentación de un show.

4.3.2.3. Ruido Intermitente: El ruido intermitente es aquel que está presente sólo durante ciertos periodos de tiempo y que son tales que la duración de cada una de estas ocurrencias es más que 5 segundos. Ejemplo: ruido producido por un comprensor de aire, o de una avenida con poco flujo vehicular.

4.3.2.4. Ruido Impulsivo: Es el ruido caracterizado por pulsos individuales de corta duración de presión sonora. La duración del ruido impulsivo suele ser menor a 1 segundo, aunque pueden ser más prolongados. Por ejemplo, el ruido producido por un disparo, una explosión en minería, vuelos de 
aeronaves rasantes militares, campanas de iglesia, entre otras.

\subsubsection{En función al tipo de actividad generadora de ruido:}

4.3.3.1. Ruido generado por el tráfico automotor.

4.3.3.2. Ruido generado por el tráfico ferroviario.

4.3.3.3. Ruido generado por el tráfico de aeronaves.

4.3.3.4. Ruido generado por plantas industriales, edificaciones y otras actividades productivas, servicios y recreativas.

\section{EFECTOS DE LA CONTAMINACIÓN SONORA}

Los efectos que produce la contaminación sonora son diversos, así tenemos:

\subsection{Efectos Auditivos}

La sordera o hipoacusia es una de las principales enfermedades profesionales propia del desarrollo industrial. En nuestro país se le ha considerado como la segunda enfermedad laboral más común después de la tendinitis (común en los digitadores).

De acuerdo a la OMS, en el mundo hay más de 120 millones de personas con deficiencias auditivas discapacitantes.

Centraremos nuestra atención en aquellos efectos sobre la audición relacionadas con el ruido ambiental y no laboral, los cuales muchas veces son considerados "normales», cuando en realidad pueden estar causando daños irreversibles.

5.1.2. Efecto Máscara: Cuando un sonido impide la percepción total o parcial de otros sonidos presentes, se dice que este sonido enmascara a los otros. Esto puede traer graves complicaciones cuando se trata del enmascaramiento de mensajes o señales de alerta y muy especialmente de la comunicación hablada. Este factor de aislamiento puede disminuir la eficacia y concentración en el trabajo, aumentando incluso el riesgo de accidentes.

5.1.3. Fatiga Auditiva: También conocido como TTS (Temporary Threshold Shift) o Cambio Temporal del Umbral Auditivo. Se trata de un déficit temporal de la sensibilidad auditiva producto de la exposición a altos niveles de ruido. Al dejar de estar expuesto al ruido, esta fatiga disminuirá gradualmente hasta recuperarse completamente. Sin embargo, si el oído es expuesto nuevamente a altos niveles de ruido antes de completarse esta recuperación, se producirá un nuevo cambio en el umbral, el cual podría hacerse permanente si estas exposiciones se tornan habituales.

5.1.4. Acufenos: Todos alguna vez en nuestra vida hemos escuchado un silbido dentro de nuestro oído. Estos sonidos se producen por la alteración del nervio auditivo que hacen escuchar un sonido interior constante que, en casos extremos puede causar ansiedad y cambios de carácter. Este efecto se le atribuye al ruido urbano.

5.1.5. Pérdida Progresiva de la Audición: Conocida también como PTS (Permanent Threshold Shift) 0 Cambio Permanente del Umbral Auditivo. Es muy habitual escuchar decir a la gente, que frecuentemente está expuesta a altos niveles de ruido, que se han «acostumbrado al ruido». Más que "acostumbramiento», lo que ocurre es que el oído no ha alcanzado a recuperarse de la fatiga auditiva o TTS, convirtiéndose paulatinamente en un cambio permanente e irreversible. La causa de esta pérdida permanente es que el ruido va matando las células auditivas, las cuales no se regeneran. Cada ser humano nace con 10.000 de estas células en cada oído. Como muchas células de nuestro organismo, éstas van muriendo en forma natural, lo que explica la sordera en los ancianos. Como esta pérdida auditiva es paulatina, las personas tienden a pensar que se han acostumbrado al ruido, lo cual es erróneo.

\subsection{Efectos No Auditivos}

En los últimos años se han relacionado una serie de patologías no auditivas producidas tanto directa como indirectamente por la exposición al ruido. Según los especialistas, dentro de las alteraciones psicológicas que produce el ruido se pueden citar las siguientes: irritabilidad, susceptibilidad exagerada, agresividad, entre otros trastornos de la personalidad.

5.2.1. Trastornos del sueño: La OMS (Organización Mundial de la Salud) recomienda que para tener un buen descanso nocturno, el ruido presente debería ser de $35 \mathrm{~dB}(\mathrm{~A})$. Para niveles de ruido mayores se comenzarían a producir perturbaciones en mayor 0 
menor grado. Por ejemplo, niveles peak de ruido muy altos (como el que provocaría una motocicleta al pasar por nuestro hogar) causarían una drástica aceleración cardíaca. Este efecto puede terminar en pacientes con hipertensión crónica.

5.2.2. Ruido y embarazo: Alrededor del quinto mes de gestación, el oído del feto se hace funcional, percibiendo los ruidos propios de su entorno inmediato, correspondiente a los del propio organismo de la madre (corazón, pulmones, voz, etc.). Experimentos realizados en poblaciones ubicadas en los entornos de aeropuertos de Japón, demostraron que los niños cuyas madres vivieron el embarazo desde el principio en dichos lugares, sufren menos alteraciones que aquellos en que la madre vivió sólo desde el quinto mes hasta el nacimiento. Se despiertan fácilmente al pasar un avión y además su peso fue inferior a la norma.

5.2.3. Aprendizaje: Los niños educados en ambientes ruidosos suelen ser menos atentos a las señales sonoras y se advierten perturbaciones en su capacidad de escuchar. En los establecimientos educacionales cercanos a vías de circulación vehicular de alto tráfico o cercanos a aeropuertos, se ha detectado un retraso en el aprendizaje de la lectura. Para lograr una buena comunicación entre el profesor y los alumnos, en una sala de clases el nivel de ruido no debiera superar los $55 \mathrm{~dB}(\mathrm{~A})$. En establecimientos educacionales cercanos a vías de alta circulación vehicular, este nivel suele ser superado ampliamente, lo que dificulta la comprensión, aumenta la falta de concentración y la baja en el rendimiento.

\section{EN CUANTO A LAS VARIABLES INDEPENDIENTES}

Las variables independientes de la presente investigación son: Regulación legal y la Contaminación sonora. Por medio de ésta sección presentaré el análisis efectuado, algunos cuadros y gráficos, que ilustren los resultados de la presente investigación.

\subsection{VARIABLE INDEPENDIENTE-REGULACIÓN LEGAL}

Como se ha establecido, el Estado reconoce constitucionalmente el derecho fundamental de las personas el vivir en un ambiente adecuado y equilibrado a su desarrollo de vida, prevista en el artículo 2.22 .

En ese sentido, El Tribunal Constitucional peruano precisó que el contenido esencial de este derecho cuenta con dos elementos: 1) el derecho a gozar de ese medio ambiente y 2) el derecho a que ese medio ambiente se preserve.

Sobre el segundo elemento el Tribunal determinó: «El derecho a la preservación de un medio ambiente sano y equilibrado entraña obligaciones ineludibles, para los poderes públicos, de mantener los bienes ambientales en las condiciones adecuadas para su disfrute. A juicio de este Tribunal, tal obligación alcanza también a los particulares, y con mayor razón a aquellos cuyas actividades económicas inciden, directa 0 indirectamente, en el medio ambiente».

Además tenemos que el Código Nacional de Tránsito sanciona el uso indiscriminado de la bocina o claxon, a las personas que cometen tal infracción como Muy Grave.

Las Municipalidades Provinciales solo pueden ejercer control sobre los vehículos de uso público, mas no sobre los de uso privado. Éstos se rigen por el Reglamento Nacional de Tránsito, en el cual se menciona el tema del ruido generado por los motores y accesorios de los vehículos de transporte.

\subsection{VARIABLE INDEPENDIENTE- CONTAMINACIÓN SONORA}

La segunda variable independiente se refiere a la situación de contaminación que se tiene en la ciudad de Juliaca producida por los medios de transporte público y privado, situación que como nos indica (Chaname Orbe, 2009) la contaminación sonora hace referencia al ruido cuando éste se considera como un contaminante, es decir, un sonido molesto que puede producir efectos fisiológicos y psicológicos nocivos para una persona o grupo de personas; teniendo como base al estudio realizado por la 
OEFA, se determina que la situación que se presenta en la actualidad en la ciudad de Juliaca, es que se produce indiscriminadamente emisiones sonoras que superan los Estándares de Calidad Ambiental(ECAS) que están establecidas según el Decreto Supremo 085-2003-PCM.

\begin{tabular}{|l|c|c|}
\hline \multirow{2}{*}{ Zonas de Aplicación } & \multicolumn{2}{|c|}{ Valores Expresados en LeqT } \\
\cline { 2 - 3 } & Horario diurno & Horario Nocturno \\
\hline Zona de Protección & $50 \mathrm{~dB}$ & $40 \mathrm{~dB}$ \\
Especial & $60 \mathrm{~dB}$ & $50 \mathrm{~dB}$ \\
\hline Zona Residencial & $70 \mathrm{~dB}$ & $60 \mathrm{~dB}$ \\
\hline Zona Comercial & $80 \mathrm{~dB}$ & $70 \mathrm{~dB}$ \\
\hline Zona Industrial & & \\
\hline
\end{tabular}

En base al cuadro anterior en comparación con el estudio de zonas de emisiones contaminantes de la Ciudad de Juliaca, se determina que se sobrepasan los Límites establecidos llegando incluso a los 80.7 $\mathrm{dB}$ en jirones del centro de la ciudad (Jr. San Román con Jr. 2 de Mayo). Existiendo un exceso de 10.7 $\mathrm{dB}$, al ubicarse esta en la Zona Comercial, determinándose en este punto la existencia plena de Contaminación Sonora.

\section{EN CUANTO A LAS VARIABLES DEPENDIENTES}

Las variables dependientes de la presente investigación son: normas emitidas por la municipalidad sobre la contaminación sonora producida por los medios de trasporte público y privado de la ciudad de Juliaca y emisiones sonoras contaminantes producidas por los medios de transporte público y privado de la ciudad de Juliaca. Veremos una a una cada una de las variables y el resultado que nos ofrece la presente investigación acompañado del análisis respectivo.

7.1. VARIABLE DEPENDIENTE- NORMAS EMITIDAS POR LAS MUNICIPALIDAD SOBRE LA CONTAMINACIÓN SONORA PRODUCIDA POR LOS MEDIOS DE TRANSPORTE PÚBLICO Y PRIVADO

La municipalidad como ente autónomo y encargado de la jurisdicción de la ciudad de Juliaca, regido por la Ley Orgánica de Municipalidades (LEY N² 27972). Esta ley establece en su artículo $46^{\circ}$ sobre la capacidad sancionadora que le faculta, señala: «las normas municipales son de carácter obligatorio y su incumplimiento acarrea las sanciones correspondientes, sin perjuicio de promover las acciones judiciales sobre las responsabilidades civiles o penales a que hubiera lugar».

Mediante el cuadro y gráfico $\mathrm{N}^{\circ} 07$ se detalla las sanciones que prevé y las impuestas por la Municipalidad de San Román-Juliaca.

\begin{tabular}{|c|c|c|c|c|c|}
\hline \multicolumn{6}{|c|}{$\begin{array}{l}\text { MUNICIPALIDAD PROVINCIAL DE SAN ROMAN - JULIACA } \\
\text { INFRACCIONES DE TRANSITO VEHICULAR }\end{array}$} \\
\hline $\mathbf{N}^{\circ}$ & INFRACCION & LEVE & GRAVE & $\begin{array}{l}\text { MUY } \\
\text { GRAVE }\end{array}$ & TOTAL \\
\hline A & $\begin{array}{l}\text { INFRACCIONES A LA } \\
\text { CONDUCCION }\end{array}$ & 42 & 15 & 8 & 65 \\
\hline B & $\begin{array}{l}\text { INFRACCIONES A LOS } \\
\text { DISPOSITIVOS DE CONTROL }\end{array}$ & 5 & 3 & 17 & 25 \\
\hline $\mathbf{C}$ & $\begin{array}{l}\text { INFRACCIONES A LA } \\
\text { SEGURIDAD }\end{array}$ & 35 & 13 & 9 & 57 \\
\hline $\mathbf{D}$ & $\begin{array}{l}\text { INFRACCIONES A LA } \\
\text { VELOCIDAD }\end{array}$ & 0 & 0 & 0 & 0 \\
\hline $\mathbf{E}$ & $\begin{array}{l}\text { INFRACCIONES AL } \\
\text { ESTACIONAMIENTO Y } \\
\text { DETENCION }\end{array}$ & 62 & 41 & 29 & 132 \\
\hline $\mathbf{F}$ & $\begin{array}{l}\text { INFRACCIONES A LA } \\
\text { DOCUMENTACION }\end{array}$ & 564 & 413 & 283 & 1260 \\
\hline G & $\begin{array}{l}\text { INFRACCIONES AL MEDIO } \\
\text { AMBIENTE }\end{array}$ & 0 & 0 & 0 & 0 \\
\hline \multicolumn{2}{|r|}{ TOTAL } & 708 & 485 & 346 & 1539 \\
\hline
\end{tabular}

*FUENTE: Unidad de tránsito/ seguridad vial MPP. De San Román-Juliaca

\subsection{VARIABLE DEPENDIENTE- EMISIONES SONORAS CONTAMINANTES PRODUCIDAS POR LOS MEDIOS DE TRANSPORTE PÚBLICO Y PRIVADO}

Las emisiones sonoras contaminantes dentro de la presente investigación se enmarca en precisar su correcta delimitación como sustenta (Andaluz Wetheicher, 2011) es el conjunto de sonidos que directa o indirectamente interfieren en el ser humano a través del sentido de la audición; a su vez estos también adquieren la denominación de ruido como advierte (Chaname Orbe, 2009) como aquel sonido no deseado, que moleste perjudique 0 afecte a la salud de las personas. Por lo que deviene, en fundamento principal la protección de la salud de la persona humana, que se encuentra enmarcado en el artículo 115 de la LEY N² 28611, Ley general del ambiente y a su vez se encuentra reconocido el artículo 2, numeral $22^{\circ}$ de nuestra Constitución Política, que señala el «Derecho a un ambiente equilibrado y adecuado para el desarrollo de la vida».

Otro punto que sustenta la existencia de emisiones contaminantes en la ciudad de Juliaca, es la 
investigación realizada por la OEFA (Organismo de Evaluación y Fiscalización Ambiental), en su estudio «Evaluación del Nivel de Ruido Ambiental en las ciudades de Lima, Callao, Maynas, Coronel Portillo, Huancayo, Huánuco, Cusco, Juliaca y Tacna en el año $2011 »$.el cual, en su tabla $N^{\circ} 7$ muestra los niveles de ruido obtenidos durante el monitoreo de 10 puntos en el distrito de Juliaca, que dan como resultado que el nivel de emisiones sonoras se encuentran entre $63.3 \mathrm{~dB}-80.7 \mathrm{~dB}$, lo que evidencia que como al ruido producido por los vehículos (motores, bocinas por el uso indiscriminado por los conductores, el parque automotor antiguo con motores extremadamente ruidosos) así como indirectamente la presencia simultánea de semáforos y policías.

\subsection{VARIABLE DEPENDIENTE- USUARIOS AFECTADOS CON LA CONTAMINACIÓN SONORA}

La contaminación sonora como tal, produce efectos álgidos y graves a nivel del ser humano, como lo demuestran los estudios realizados, tanto por la OMS así como validados a nivel mundial, que nos indican que con la sola exposición a niveles superiores a los estandarizados generaran que la población sea afectada directa 0 indirectamente. Así contribuye también Fonseca (Fonseca Tapia, 2010) la contaminación acústica perturba las distintas actividades comunitarias, interfiriendo la comunicación hablada, base esta de la convivencia humana, perturbando el sueño, el descanso y la relajación, impidiendo la concentración y el aprendizaje, y lo que es más grave, creando estados de cansancio y tensión que pueden degenerar en enfermedades de tipo nervioso y cardiovascular.

Ahora bien de los estudios realizados por la OEFA, definitivamente se establece que en la ciudad de Juliaca Existen emisiones sonoras contaminantes, en diversas partes de la ciudad, por lo que sería renuente y vacuo hacer una sobre investigación de los afectados, puesto que como está definido con el aporte mencionado, este definió que enfermedades produce esta contaminación, es así que simplemente nos aunamos a las expuestas por la OMS que refiere, (Organizacion Mundial de la Salud, 2013) los efectos que produce la contaminación sonora son de dos tipos, los cuales son daños auditivos como el Efecto Máscara, la Fatiga Auditiva, los Acúfenos, o la más producida Pérdida Progresiva de la Audición, todas estas afecciones se producirán paulatinamente, son irreversibles, y la causa de esta pérdida permanente es que el ruido que va matando las células auditivas, las cuales no se regeneran. Ahora bien dentro de los daños no auditivos se tiene como principal afección al Trastorno del sueño, que como lo sostiene la OMS (Organizacion Mundial de la Salud, 2013) recomienda que para tener un buen descanso nocturno, el ruido presente, no debería exceder de $35 \mathrm{~dB}$. Según este estudio la presencia de niveles de ruido mayores comenzarían a producir perturbaciones en mayor 0 menor grado, por lo que en relación al estudio que Realizo la OEFA, se tiene que en la ciudad de Juliaca se tiene emisiones de picos que sobrepasan los $80 \mathrm{~dB}$, por lo que se deduce que la población en general tiene un riesgo 0 asociación a este efecto.

\section{CONCLUSIONES}

La legislación en nuestro país es variada, dispersa y frecuentemente confusa. En ese sentido, se establecen como normas de protección del medio ambiente y la salud de la persona, en primer orden tenemos: la Constitución Política del Perú que reconoce que toda persona tiene el derecho a vivir en un ambiente adecuado y equilibrado, en el cual pueda desenvolverse, así mismo se tiene el Reglamento Nacional de Vehículos, el cual regula, a nivel nacional pero de manera vacua las reglamentaciones que se debe tener por parte de los vehículos tanto privados como públicos; en segundo orden se tiene las Ordenanzas Municipales, sin embargo, Las Municipalidades Provinciales solo pueden ejercer control sobre los vehículos de uso público, mas no sobre los de uso privado. Éstos se rigen por el Reglamento Nacional de Tránsito, en el cual se menciona el tema del ruido generado por los motores y accesorios de los vehículos de transporte; y en tercer lugar tenemos al Ministerio del Ambiente, el cual regula de manera específica y adecuada a través del Decreto Supremo 085-2003-PCM, definiendo los estándares de calidad ambiental, delimitando de manera exacta y con límites cada una de las zonas de una población y así evitar la contaminación sonora.

Las emisiones contaminantes en la ciudad de Juliaca sobrepasan los $80 \mathrm{~dB}$, por lo que existe una profusa contaminación sonoras, puesto que los niveles 
permisibles establecidos oscilan entre los $50 \mathrm{~dB}$. En la zona media, es decir entre residencial y comercial, se tiene como límites permisibles como máximo los $60 \mathrm{~dB}$.

La contaminación sonora a través del ruido como agente contaminante produce una serie de daños tanto físicos como psicológicos con efectos negativos en la población de manera general a través de daños auditivos: como el Efecto Máscara, la Fatiga Auditiva, los Acúfenos o la Pérdida Progresiva de la Audición, afecciones que se producirán paulatinamente y son irreversibles. Por otro lado, los daños no auditivos, como el stress, ansiedad, irritabilidad, etc., que causan perjuicio directo en la persona al afectar directamente sus relaciones sociales, familiares y laborales.

Debe ser función de la Política Nacional en nuestro país, la Protección de la Persona Humana, especialmente en materia ambiental a través del Ministerio de Ambiente, teniendo presente en todo momento la noción de desarrollo sostenible, el cual parte del reconocimiento del derecho que tiene todo ciudadano a movilizarse en un ambiente sano y que no le cause perjuicio, subrayando, que el Estado tiene la obligación de asegurar las condiciones propicias para que ese derecho pueda ser ejercitado adecuadamente, lo que implica, entre muchas otras cosas, la toma de acciones tendientes a integrar los planes sobre transporte, desarrollo urbano y los relativos a la protección de las personas y el medio ambiente.

\section{RECOMENDACIONES}

Es necesario que el Gobierno Nacional y entes de Gobierno Municipal, establezcan dentro de su esquema organizacional la integración de comisiones técnicas encargadas de elaborar y desarrollar los Planes de Gestión Ambiental respecto a la contaminación sonora que producen los medios de transporte público y privado, a través de un sistema de control de ruidos provocado por el parque automotor ya que este es el principal agente emisor de ruidos contaminantes.

Se debe promulgar una Norma Nacional sobre la Contaminación Sonora, dejando a las Municipalidades, planearla dirigirla, reglamentarla, así como establecer prohibiciones y sanciones específicas, a fin de garantizar la protección de un medio ambiente sano y la salud de sus habitantes, dentro de su jurisdicción.

Las políticas urbanas de transporte no pueden ser exitosas sin la cooperación integral de todos los ciudadanos, esta cooperación estará asegurada si se pone a conocimiento de éstos, de la manera más clara posible, el objetivo de cualquier iniciativa. Por ello es necesario llevar a cabo campañas que informen a las personas de las terribles consecuencias que acarrea la contaminación sonora, que en este caso está determinada por el incremento del parque automotor en las áreas urbanas, atentando a su salud y bienestar. Estas campañas deben estar dirigidas a incentivar el uso del transporte público y de los vehículos privados de manera correcta y consciente de manejo, que harán que el nivel de contaminación disminuya. Finalmente hay que poner especial atención en la educación de los más pequeños, implementando la enseñanza de las políticas relativas al sistema de transporte, en los planes curriculares de las escuelas.

\section{BIBLIOGRAFÍA}

Andaluz Wetheicher, C. (2011). Manual de Derecho Ambiental (Primera ed.). Lima, Peru: Iustitia. Basombrío Iglesias, C. (2005). Reflexiones sobre la experiencia de activistas e intelectuales de sociedad civil en la función pública. Lima, Peru: Instituto de Estudios Peruanos.

Bernal Garcia, M. (2011). Normas de protección del medio ambiente. . Lima: Jurista Editores. Brañes, R. (2000). Manual de Derecho Ambiental Mexicano. Mexico: Fondo de Cultura Económica. Cafferatta, N. A. (2004). Introducción al Derecho Ambiental. Mexico : Instituto Nacional de Ecología. Carhuatocto Sandoval, H. (2009). Guía de Derecho Ambiental. Lima, Peru: Jurista Editores.

Chaname Orbe, R. (2009). Diccionario de terminos y conceptos (Primera ed.). Rodhas.

Flores, E., Dominguez, R., \& Sanchez, J. M. (1998). Contaminacion Acustica. Madrid: Adrus.

Fonseca Tapia, C. (2010). Derecho Ambiental (Primera ed.). Lima, Peru: Adrus.

OEFA. (2011). Evaluación Rápida Del Nivel De Ruido Ambiental en las Ciudades de Lima, Callao, Maynas, Coronel Portillo, Huancayo, Huánuco, Cusco, Juliacay Tacna. Lima: Dirección de Evaluación. 
Organizacion Mundial de la Salud, M. (2013). Estudio Sobre la Contaminacion Sonora. Lima.

Santander Mejía, E. (2002). Instituciones de Derecho Ambiental. Bogota, Colombia: ECOE.

\section{REFERENCIAS BIBLIOGRÁFICAS}

Andaluz Wetheicher, Carlos (2011 (Flores, Dominguez, \& Sanchez, 1998), «Manual de DerechoAmbiental», primera Edición, Lima: Editorial lustitia.

Basombrío Iglesias, Carlos. (2005). «Reflexiones Sobre la Experiencia de Activistas e Intelectuales de Sociedad Civil en la Función Pública». Lima: Instituto de Estudios Peruanos.

CAFFERATTA, Néstor A. (2004). «Introducción alDerecho Ambiental», México, Instituto Nacional deEcología.

CARHUATOCTO SANDOVAL Henry (2009). «Guía de Derecho Ambiental» Jurista Editores.

CARRUITEROLECCA, Francisco (2006). "Derechodel Medio Ambiente», Lima: Editorial Estudio.

CHANAME ORBE, Raúl (2009). «Diccionario de Términos y Conceptos» Primera Edición, Editorial Rodhas.

FONSECA TAPIA, Cesar A. (2010). "Derecho Ambiental», Primera Edición, Editorial Adrus.

FLORES, E. ; DOMÍNGUEZ, R. ;SÁNCHEZ, J. M.1998. ContaminaciónAcústica, España,jesusma@caymasa.es.] [Consulta: 2013-08].

\section{LEGISLACIÓN}

* LEY GENERAL DEL AMBIENTE - LEY N ${ }^{\circ}$ 28611

* LeY mARCO DEL SISTEMA nAcIONAL DE GESTIÓN AMBIENTAL - LEY N 28245

* REgLAMENTO DE LA LEY MARCO DEL SISTEMANACIONAL DE GESTIÓN AMBIENTAL-DECRETO SUPREMO No 008 2005 - PCM

* LEY DE CREACIÓN, ORGANIZACIÓN Y FUNCIONES DEL MINISTERIO DEL
AMBIENTE-DECRETO LEGISLATIVO No 1013

Decreto Supremo N ${ }^{\circ}$ 085-2003-PCM (30/Oct/ 03) «Aprueban el Reglamento de Estándares Nacionales de Calidad Ambiental para Ruido»

\section{INFOGRAFÍA}

DOMÍNGUEZ RODIÑO, Eloy Flores y SÁNCHEZ GONZÁLEZ Jesús María (2013-10-02) http:// www.sorolls.org/docs/CA_monografias.htm. [Consulta: 2013-10].

FLORES, E. ; DOMÍNGUEZ, R. ; SÁNCHEZ, J. M. 1998. Contaminación Acústica, España, jesusma@caymasa.es. [Consulta: 2013-11].

WIKIPEDIA http://es.wikipedia.org/wiki/ Transporte_privado. [Consulta: 2013-10]. http://www1.ceit.es/Asignaturas/Ecologia/Hipertexto/ 10CAtm1/320CoSon.htm. [Consulta: 2013-10].

http://www.todoelderecho.com/Apuntes/Ambiental/ Apuntes/CONTAMINACION\%20SONORA.htm. [Consulta: 2013-11].

http://ruido_peru.pe.tripod.com/ [Consulta: 2013-12]. http://www.conam.gob.pe/modulos/home/ queeselconam.asp [Consulta: 2013-12].

MINISTERIO DEL AMBIENTE. (s.f.). Recuperado el 12 de 04 de 2014, de www.minam.gob.pe. 